\title{
Finite element analysis of hybrid energy harvesting of piezoelectric and electromagnetic
}

\author{
Muhammad Ammar Faris Muhammad Yazid ${ }^{1}$, Norlida Jamil ${ }^{1}$, Nik Nurul Husna Muhmed \\ Razali $^{1}$, and Ahmad Razlan Yusoff ${ }^{1, *}$ \\ ${ }^{1}$ Faculty of Manufacturing Engineering, Universiti Malaysia Pahang, 26600, Pekan, Pahang, Malaysia
}

\begin{abstract}
Harvesting energy from ambient vibrations is a highly required method because of the wide range of available sources that produce vibration energy application from industrial machinery to human motion application. In this paper, the implementation of harvesting energy from two technologies to form a hybrid energy harvester system was analyzed. These two technologies involve the piezoelectric harvesting energy and the electromagnetic harvesting energy. A finite element model was developed using the Ansys software with the harmonic analysis solver to analyze and examine hybrid harvesting energy system. Both power output generated from the magnet and the piezoelectric is then combined to form one unit of energy. Further, it was found that the result shows the system generate the maximum power output of $14.85 \mu \mathrm{W}$ from $100 \mathrm{~Hz}, 4.905 \mathrm{~m} / \mathrm{s} 2$, and 0.6 $\mathrm{cm} 3$ for resonance frequency, acceleration, and the volume respectively from the optimal energy harvester design. Normalized Power Density (NPD) result of $10.29 \mathrm{kgs} / \mathrm{m} 3$ comparable with other literature also can be used in energy harvesting system for vibration application.
\end{abstract}

\section{Introduction}

The term energy harvesting refers to the generation of energy from sources such as ambient temperature, vibration or air flow. A typical energy harvesting system starts with an energy collector or transducer device and depends on the type of energy one is trying to convert. These are typically solar or photovoltaic cells for light energy, piezoelectric for pressure, kinetic for movement, inductive for rotational or motion, thermoelectric for heat or temperature differential, and electromagnetic. The energy harvesting system is quiet significant as it has the potential to replace the batteries for small, low power electronic devices. Besides, the stored electrical energy can then be used by various sensor nodes for applications such as sensing, actuating, or sending wireless signals.

The most common types of energy harvesting are photonic, thermal, and vibration. Common photonic harvesters rely on solar energy drawn with the use of photovoltaic. However, according to Kwang [1], solar cells are not $100 \%$ efficient because some spectrums of the sunlight are being reflected, some are too weak to create electricity and some create heat energy instead of electricity. Thermoelectric devices used for energy

\footnotetext{
* Corresponding author: razlan@ump.edu.my
} 
harvesting convert thermal activity into electricity. They do not require generators or pumps or fluids, and do not require abundant amounts of materials to build. But it has low efficiency as compared to other mechanical generators in which thermoelectric power generators generate less electric power for the same heat flow [2].

The method of the hybrid has become more popular for the development of the energy harvesting devices. Hybrid energy harvesting devices combine more than one of the energy scavenger systems to create a more efficient unit. El-Hami et al. [3] proposed design of a cantilever beam with two magnets attached to a C-shaped core was able to increase the natural frequency of the system up to $322 \mathrm{~Hz}$. However, the frequency produce is very high from the goal to obtain a system with a low frequency as less than $30 \mathrm{~Hz}$ to capture vibrations from the ambient. Three years later, Duffy et al. [4] has developed an electromagnetic energy harvester that consists of two fixed magnets at each end of the device which act as the springs. Through this design, the research done was integrated in a shoe sole with the intention of collecting energy from human motion. Nevertheless, this device is relatively large in size and interferes with the human motion. Familiar mechanism of energy harvester is presented by Hassan et al. [5]. It is found that the induced voltage is proportional to the increment of the number of turns and the length of beams. Moreover, Soliman et al. [6] presented a mechanical stopper that is in the range of motion of a cantilever beam in order to increase the range of operation. When the beam is contact with the stopper, the stiffness of the beam would rises and causing the alteration on the resonance frequency of the system. In 2001, White et al. [7] reported that alternative way of scavenging the energy using the piezoelectric. By applying stainless steel tapered base with two layers of piezoelectric film printed on both sides, the deflection of the piezoelectric layers can be maximized and apparently increase the power output. Besides, Yu et al. [8] reported that more power output can be generated than a stand-alone piezoelectric or electromagnetic device, and the output voltage is increased by using the mems pzt cantilever array architecture. This configuration has also been implemented by Roundy [9]. However this configuration would only make the area of the beam near the clamped end receive the maximum strain and causing an uneven strain distribution along the cantilever beam. Therefore, Challa et al. [10] has proposed configuration consisted of a piezoelectric cantilever beam with a tip mass and magnets on both sides of the free end.

In this paper, a model of hybrid energy harvesting system made of piezoelectric and electromagnetic will be presented by using the harmonic analysis implemented in the Ansys software. The hybrid energy harvesting system will also be analyze several number of sample consist with different width and length of bimorph piezoelectric beam to produce power from the deflection of the beam.

\section{Analytical design and simulation}

\subsection{Calculation of piezoelectric power output}

The output power produce from the bimorph cantilever beam of piezoelectric with different geometrical dimension using Finite Element Analysis (FEA) can be obtained by deriving from the constitutive equations for a piezoelectric material as follows:

$$
\begin{aligned}
& \delta=\frac{\sigma}{Y}+d E \\
& D=\varepsilon E+d \sigma
\end{aligned}
$$


Where $\delta$ is mechanical strain, $\sigma$ is mechanical stress, $\mathrm{Y}$ is the modulus of elasticity, $\mathrm{d}$ is the piezoelectric strain coefficient, $\mathrm{E}$ is the electric field, $\mathrm{D}$ is electric displacement, and $\varepsilon$ is the dielectric constant of the piezoelectric material. According to Roundy [9], by assuming the driving frequency is not corresponding with the natural frequency, he suggested that the voltage magnitude that is transmitted to the load for a piezoelectric beam can be given as follows:

$$
V=\frac{-\frac{j \omega Y_{c} d t_{c} b^{*}}{\varepsilon}}{\left[\frac{1}{R C_{p}} \omega_{n}^{2}-\left(\frac{1}{R C_{p}}+2 \zeta \omega_{n}\right) \omega^{2}\right]+j \omega\left[\omega_{n}^{2}\left(1+k^{2}\right)+\frac{2 \zeta \omega_{n}}{R C_{p}}-\omega^{2}\right]} A_{i n}^{2}
$$

Where $\mathrm{V}$ is the generated voltage from the piezoelectric material, $\omega$ is the driving frequency, $Y c$ is Young's Modulus for a piezoelectric material, $\mathrm{d}$ is the piezoelectric strain coefficient, $t c$ is the thickness of the piezoelectric material, $b^{*}$ is strain related to vertical displacement of the beam, $\varepsilon$ is the dielectric constant of the piezoelectric material, $\mathrm{R}$ is the load resistance, $C p$ is the capacitance of the piezoelectric device, $\omega n$ is the natural frequency of the system, $\zeta$ is the mechanical damping ratio, $\mathrm{k}$ is a coupling coefficient, and Ain is the magnitude of the input acceleration. Meanwhile as for the power transferred to the load, the general equation of V2/R can simply be use. Equation 3 can be further simplified if the natural frequency $\omega \mathrm{n}$ is assumed that it is equivalent to the driving frequency, $\omega$. This is because the generated power is maximized when the vibration frequency is equal to the resonant frequency. Thus, the natural frequency and the environment frequency must be very similar. The power output, $\mathrm{P}$ of the beam can then be formulated as follows:

$$
P=\frac{1}{2 \omega_{n}^{2}} \frac{R C_{p}^{2}\left(\frac{d t_{c} \sigma_{\text {ave }}}{\varepsilon Z_{t}}\right)^{2}}{\left(4 \zeta^{2}+k^{4}\right)\left(R C_{p} \omega_{n}\right)^{2}+4 \zeta k^{2}\left(R C_{p} \omega_{n}\right)+(2 \zeta)^{2}}
$$

\subsection{Calculation of electromagnet output}

The output power produce from the electromagnetic using the finite element analysis (FEA) can be obtained by using the manipulation of resonant technique. Electromagnetic coils such as solenoids, inductors, electromagnets, and electric motors often time required high-current and high-frequency operation. Figure 1 shows the example of the amplifier circuit that implements the resonant technique. First of all, the basic equation to get the power can be simply by using the Equation 5 as follows where $\mathrm{P}$ is the power output, $\mathrm{I}$ is the current and $\mathrm{V}$, is the voltage. However, the magnitudes of the voltage are needed to be acquired from the simulation first. Meanwhile, the current flow on the circuit can be simply calculated by using the current equation at the specified frequency by using the Equation 6 . It shows that capacitor reactance, $\mathrm{C}$ whereby at a given resonant frequency, $\mathrm{f}$ can be calculated by using the Equation 7 with the properties of the capacitance given in the Table 3 later. 


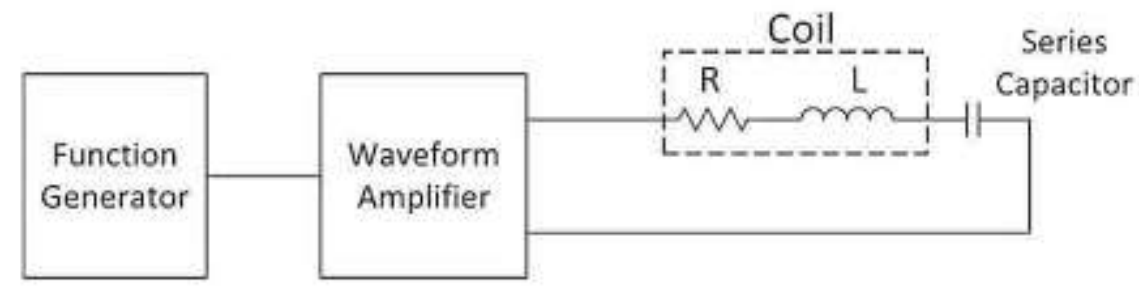

Fig. 1. Waveform amplifier drives current through the coil at resonance.

$$
\begin{gathered}
P=I V \\
I=V \omega C \\
C=\frac{1}{(2 \pi f)^{2}}
\end{gathered}
$$

\subsection{Finite element analysis}

Initially, the proposed design will be model via SolidWork software. Then the created model will be import into the Ansys software to perform the finite element simulation. A three dimensional harmonic analysis was run to determine both the deflection and the voltage output of the piezoelectric material. This study will focus on the design of hybrid harvester that able to scavenge the ambient energy and operate at low frequencies that are normally between 10-125 Hz. The proposed design comprised of the piezoelectric material that is mounted as bimorph cantilever beam. This design incorporates a piezoelectric cantilever beam with a magnet attached at the tip of the beam as shown in the Figure 2 . The piezoelectric layer is fixed at the points of contact with the mount and as the system vibrates, it will experience the deflection. Therefore, the magnet that is attached at the tip of the piezoelectric beam will also move vertically inducing a voltage inside the copper coil through Faraday's law of induction. Hence, both piezoelectric and magnet will actively contribute to the energy harvesting process.

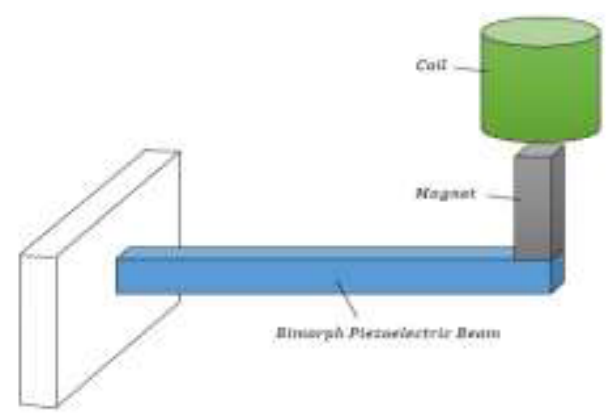

Fig. 2. Piezo-electromagnetic design concept.

The finite element analysis was executed with several numbers of samples that is made up with a variance of dimension for the cantilever beam. The variable parameter of dimension comprise of the length and the width of the beam. Whereas the thickness of the 
beam and load applied to the system were set to be constant. The sample and its dimension are listed in the Table 1.

Table 1. List of dimension for each cantilever beam sample.

\begin{tabular}{|c|c|c|c|}
\hline Beam & Length $(\mathbf{m m})$ & Width $(\mathbf{m m})$ & Thickness $(\mathbf{m m})$ \\
\hline A & 16.5 & 6.35 & 0.51 \\
\hline B & 36.5 & 12.7 & 0.51 \\
\hline C & 36.5 & 19.05 & 0.51 \\
\hline D & 36.5 & 6.35 & 0.51 \\
\hline E & 36.5 & 12.7 & 0.51 \\
\hline F & 36.5 & 19.05 & 0.51 \\
\hline
\end{tabular}

Two types of material used are magnet and piezoelectric materials. The designs of this energy harvesting consist of Neodymium Iron Boron (NdFeB) magnets. Neodymium magnets are commonly used in the harvesting application as it can be considered as one of the strongest permanent magnets that can be made. Based on the basic operation of magnet and coils, an oscillation movement of the coil through the magnet provides the change in magnetic field and induces voltage in the coil. Coil use for the design is Tinned Copper wire (MIL-W-3861 TYPE S) type. This tinned copper is electrolytic, soft drawn and annealed that allows to be easily formed. The piezoelectric material will be using the PZT$5 \mathrm{~A} 4 \mathrm{E}$. There will be two layer of piezoelectric sandwich together with a layer of shim in between to form the bimorph beam. The shim layer will be made of the Brass material.

The full harmonic solver was used with element type Solid 226 with KEYOPT (1) = 1001 to activate the electromechanical coupling. It is a coupled field 20 nodes brick element. The structure is excited by a sinusoidal wave with an acceleration magnitude of $4.905 \mathrm{~m} / \mathrm{s} 2$ and frequency range of $0-500 \mathrm{~Hz}$. Because the tip mass is located of fcenter at the free end of the cantilever beam, it introduces a torque and force which cause the material to bend. Prior to the simulation, both material properties of piezoelectric and the brass are defined. These properties can be found in Table 2 .

Table 2. Mechanical and electrical properties used in the FEA.

\begin{tabular}{|c|c|c|}
\hline Parameters & Piezoelectric Material & Brass Shim \\
\hline Young's modulus (GPa) & 66 & 117 \\
\hline Yield Stress $(\mathrm{MPa})$ & 24 & 200 \\
\hline Maximum deflection $(\mu \mathrm{m})$ & 300 & - \\
\hline Poisson's ratio & 0.31 & 0.324 \\
\hline Density $\left(\mathrm{kg} / \mathrm{m}^{3}\right)$ & 7800 & 7165 \\
\hline Relative dielectric constant & 1800 & - \\
\hline $\mathrm{d} 31(\mathrm{~m} / \mathrm{V})$ & $-190 \times 10^{-12}$ & - \\
\hline
\end{tabular}

In general, meshing influences the accuracy of an analysis. Number of mesh element of the system is 11528 with default setting by system, as illustrated in Figure 3(a). Also, ANSYS also provides the user with interface tools to ease the process of assigning 
boundary conditions. In Figure 3(b), one end layer of the cantilever beam is fixed at three coordinates ( $\mathrm{x}, \mathrm{y}$, and $\mathrm{z}$ ). Meanwhile, a concentrated pressure is applied at the other free end of the cantilever beam. The load is defined by placing geometry with predefined mass properties of $4.1 \mathrm{~g}$. Because the tip mass is located off-center at the free end of the cantilever beam, it introduces a torque and force which cause the material to bend. The structure is excited by a sinusoidal wave with an acceleration of $4.905 \mathrm{~m} / \mathrm{s} 2$ with the frequency of $0-500 \mathrm{~Hz}$.

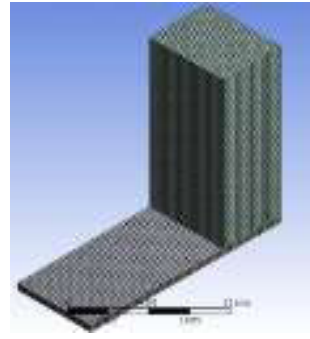

(a) Meshing the geometry

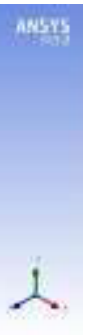

(b) The fixed support area is selected at the free end of the cantilever beam

Fig. 3. Finite element analysis setting.

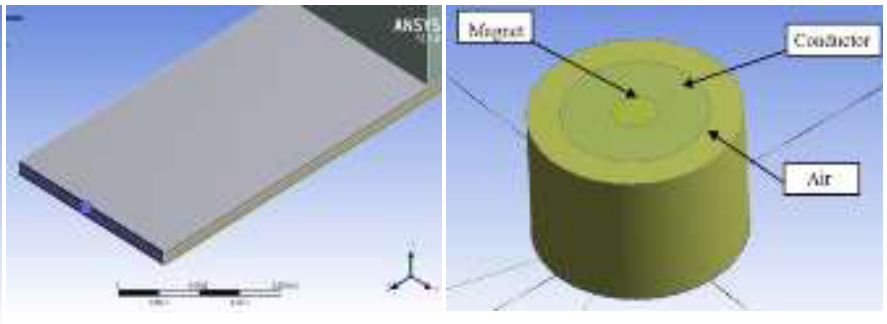

(c) Magnetic model in Ansys Workbench

\subsection{FEA: magnetostatic analysis}

Figure 3(c) shows the geometry of a magnet model that was created and was analyses via magneto static analysis. There is a magnet that moves in the vertical direction and a stationary multi turn coil, surrounded by air. However, due to the limitation of this software, only the static analysis can only be performing to make the analysis. This geometry was then meshed with 89556 triangular elements. The values of the properties that where inputted are given in Table 3 . To properly model the magnetic field around the magnet and coil, the air around the magnet and the coil must also be modeled and meshed in Ansys.

Table 3. Material property of magnet.

\begin{tabular}{|c|c|}
\hline Property & Value \\
\hline Multi Turn Coil & 100 Turns \\
\hline Coil Material & Copper \\
\hline Wire Gauge Diameter & $0.0897 \mathrm{~mm}$ \\
\hline
\end{tabular}

\section{Results and discussion}

\subsection{Piezoelectric beam: analysis result}

They are six sample of cantilever beam with the difference dimension were proposed to be analyzed. Therefore, it can be summarizes the difference between each beam according to the total volume and the total mass as follows. It clearly shows that cantilever beams with the high amount of volume are mostly come from the beam with the length of $36.5 \mathrm{~mm}$. Beam $\mathrm{F}$ leading the volume amount with the $0.355 \mathrm{~cm} 3$. Meanwhile, Beam A has the least 
volume amount with the $0.0534 \mathrm{~cm} 3$. Analysis of this hybrid energy harvester can be described by analytical and finite element analysis. The results of this analysis are summarized in Table 4.

Table 4. Experimental result for hybrid energy harvester.

\begin{tabular}{|c|c|c|c|c|c|}
\hline Beam & $\begin{array}{c}\text { Max Stress of } \\
\text { Beam (MPa) }\end{array}$ & $\begin{array}{c}\text { Deflection of } \\
\text { Beam (mm) }\end{array}$ & $\begin{array}{c}\text { Resonance } \\
\text { Frequency of } \\
\text { Beam (Hz) }\end{array}$ & $\begin{array}{c}\text { Voltage } \\
\text { Output of } \\
\text { Beam (V) }\end{array}$ & $\begin{array}{c}\text { Power } \\
\text { Output of } \\
\text { Beam ( } \boldsymbol{\mu W})\end{array}$ \\
\hline A & 0.49 & 0.029 & 150 & 8.55 & 14.9 \\
\hline B & 0.72 & 0.042 & 200 & 9.04 & 16.9 \\
\hline C & 0.24 & 0.047 & 250 & 2.42 & 1.50 \\
\hline D & 0.11 & 0.058 & 300 & 3.04 & 2.00 \\
\hline F & 0.26 & 0.059 & 100 & 8.24 & 14.0 \\
\hline
\end{tabular}

The force applied on the piezoelectric beam would generate a stress. However, the stresses in the beam should be low enough so that it will not break or crack from the input acceleration. It is observed from the simulation that even the beam with the highest amount of stress applied (Beam F); will not break under their maximum stress as presented in the Table 5 which within the strength constraints of $\sigma=24 \mathrm{MPa}$. Moreover, from the data tabulated in the Table 5, it is shows that the beam $\mathrm{D}$ produces maximum deflection value of $0.11047 \mathrm{~mm}$. In contrary, the beam $\mathrm{C}$ has the minimum deflection with the value of 0.0124 $\mathrm{mm}$. The consequent results obtained from the deflection effect of each beam were also affecting the natural frequency range produce on each system. The minimum and maximum resonance frequency of the system occur at $50 \mathrm{~Hz}$ (beam D) and $450 \mathrm{~Hz}$ (beam F) respectively. Since the aim of analysis is scavenging the energy at low range of frequency as possible, thus, only beam $\mathrm{A}, \mathrm{D}$ and $\mathrm{E}$ are satisfied the requirement with the frequency of $150 \mathrm{~Hz}, 50 \mathrm{~Hz}$, and $100 \mathrm{~Hz}$ respectively.

Using Equation 3 and 4, the voltage and power generated of each beam are calculated. Beam B from length $16.5 \mathrm{~mm}$ conducted highest voltage output with $9.04 \mathrm{mV}$. Meanwhile, the beams from width of $12.7 \mathrm{~mm}$ have generated the most voltage output to the system. Therefore, it can be deduced that the effective range of the width for this particular geometry with the constant material property and the load applied evolve around the range of $12.7 \mathrm{~mm}$. Moreover, it shows the similar trend for power output determination. Highest magnitude of voltage comes from the width of $12.7 \mathrm{~mm}$. For the length of $16.5 \mathrm{~mm}$, the maximum power output is $16.9 \mu \mathrm{W}$. While for the length of $36.5 \mathrm{~mm}$ generate slightly lower amount of the power output with $14.0 \mu \mathrm{W}$. However, considering the validity of the low range frequency as discussed before, only beam A and E (as illustrated in Figure 4); were approve to be implemented with the highest magnitude of the power output generated from the beam A, $14.9 \mu \mathrm{W}$. 


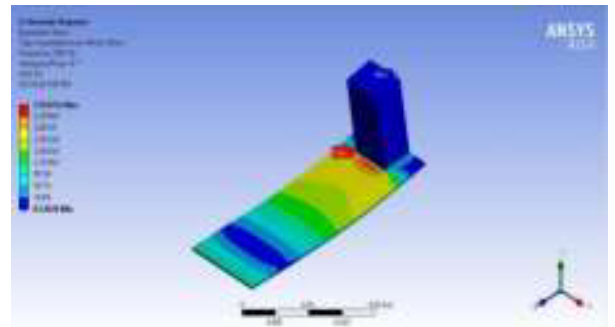

(a) Stress distribution of beam

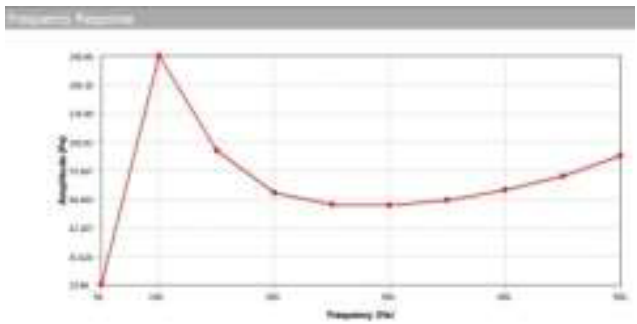

(b) Resonance frequency

Fig. 4. Beam E finite element analysis result.

\subsection{Voltage and power output}

Figure 5(a) shows the cross section geometry of the total magnetic flux density produced from the electromagnetic analysis. The value of the total magnetic flux density produced is $1.52 \mathrm{~T}$. Meanwhile, the voltage output generated from the electromagnetic was showed in the Figure 5(b). The highest voltage output that can be produced is $7.25 \mathrm{mV}$ is produced. Therefore, by using the Equation 5, the power output of the system performed at the frequency of $100 \mathrm{~Hz}$ is calculated with the value of $0.85 \mu \mathrm{W}$.

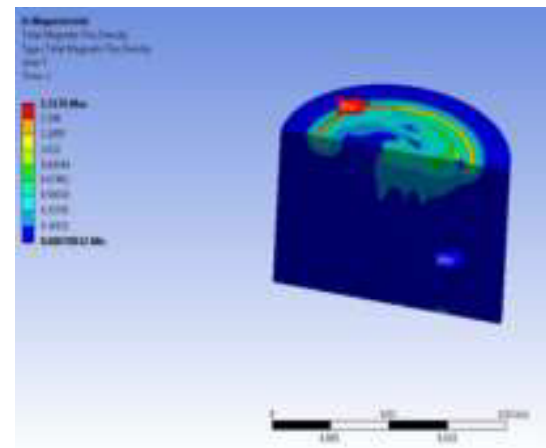

(a)

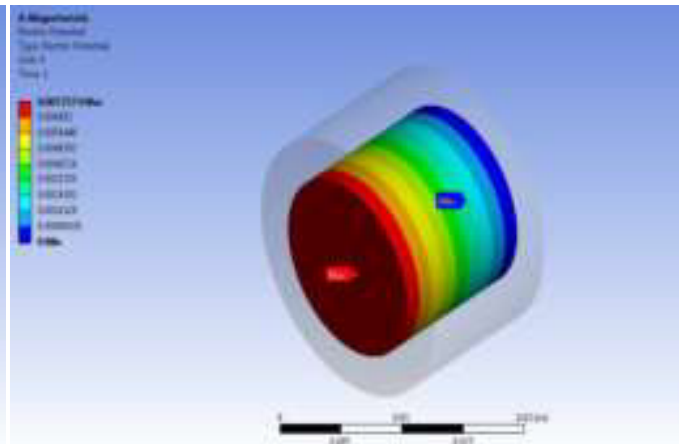

(b)

Fig. 5. Electromagnetic analysis.

The highest amount of power output comes from the bimorph piezoelectric cantilever beam $\mathrm{E}$ with the value of $14.0 \mu \mathrm{W}$ at the frequency range of $100 \mathrm{~Hz}$. Meanwhile, the electromagnetic harvesting technology produced the output power of $0.85 \mu \mathrm{W}$. Generally, power output generated from both harvesting technologies was combined to form one power output for the system through the series circuit. By having both systems running under the same range of frequency which is $100 \mathrm{~Hz}$, the hybrid energy harvesting system is able to produce the power output up to $14.85 \mu \mathrm{W}$.

\subsection{Comparison of normalized power density}

Currently in the field of energy harvesting there is not one single unified method for comparing the effectiveness of harvesters due to the large variations in the data that is presented in published works. This means some assumptions and calculations have to be made from the data provided. In 2007, Beeby et al. [11] has derived a measure for normalized power density (NPD) that was used only as an indication of relative 
performance. The measurement is described as in the Equation 8 where $\mathrm{P}$ is the peak power output, $\mathrm{A}$ is the base acceleration, and $\mathrm{V}$ is the volume of the harvester.

$$
\mathrm{NPD}=\frac{P}{A^{2} V}
$$

Table 5 compares some available harvesters with their respective NPD information. From presented data, it shows a few harvesters that have higher NPD values. In comparison, [12] has the least value of NPD with 0.09 while the study made by Beeby et al. [11] has the highest amount of value with 883 . However, the calculation made was using only the volume of the magnets. If the full volume of the harvester which includes the bulk volume of the magnets, the tungsten mass used to tune the frequency, and the cantilever beam was to be considered, this value would drop to around 70 .

Table 5. Comparison with other published energy harvester.

\begin{tabular}{|c|c|c|c|c|c|}
\hline $\begin{array}{c}\text { Energy } \\
\text { Harvesters }\end{array}$ & $\begin{array}{l}\text { Frequency } \\
(\mathbf{H z})\end{array}$ & $\begin{array}{l}\text { Acceleration } \\
\left(\mathrm{m} / \mathrm{s}^{2}\right)\end{array}$ & $\underset{\left(\mathrm{cm}^{3}\right)}{\text { Volume }}$ & $\begin{array}{c}\text { Power } \\
(\mu W)\end{array}$ & $\underset{\left(\mathrm{kgs} / \mathrm{m}^{3}\right)}{\mathrm{NPD}}$ \\
\hline $\begin{array}{l}\text { Current } \\
\text { study }\end{array}$ & 100 & 4.905 & 0.06 & 14.85 & 10.29 \\
\hline $\begin{array}{c}\text { Beeby et al. } \\
{[11]}\end{array}$ & 52 & 0.589 & 0.15 & 46 & 883.97 \\
\hline $\begin{array}{c}\text { Roundy } \\
\text { [9] }\end{array}$ & 157 & 2.5 & 1 & 375 & 60.00 \\
\hline $\begin{array}{c}\text { Karami } \\
{[13]} \\
\end{array}$ & 8 & 1.18 & 8.6 & 110 & 9.19 \\
\hline $\begin{array}{c}\text { White et al. } \\
{[7]}\end{array}$ & 80 & 2.3 & 0.125 & 2.1 & 3.18 \\
\hline $\begin{array}{c}\text { Ching et al. } \\
{[12]}\end{array}$ & 110 & 95.5 & 1 & 830 & 0.09 \\
\hline
\end{tabular}

Besides, Roundy [9] has produced one of the highest amounts of NPD value and power output; however it compensate with the higher range of operating frequency. Although Karami [13] designed a harvester that operates at $8 \mathrm{~Hz}$ but it is large in size with the beam dimensions, $8.6 \mathrm{~cm} 3$ whereas the hybrid PE energy harvesting system has the least amount of volume of $0.06 \mathrm{~cm} 3$. In terms of power output, White et al. [7] has the least amount of power output produced with $2.1 \mu \mathrm{W}$ while Ching et al. [12] obtained the highest amount of power output in compare to the others. Therefore, in comparison hybrid PE energy harvesting has the advantage in terms of the dimension which relatively small and can produce the acceptable amount of power output at a low range frequency.

\section{Conclusions}

The performance of the hybrid harvesting energy has been investigated. From the proposed design, all beam has passed the allowable stress as it well within the strength constraints of $\sigma=24 \mathrm{MPa}$. The maximum power output is generated from bimorph piezoelectric beam comes from the beam A with the magnitude of $16.9 \mu \mathrm{W}$. However, due to the objective of this study in which to scavenge the energy at the lower range of frequency, therefore the 
beam $\mathrm{E}$ has been selected as it has the least resonance frequency of $100 \mathrm{~Hz}$ and can produce up to is $14.0 \mu \mathrm{W}$. Meanwhile, the electromagnetic harvesting technology produced the output power of $0.85 \mu \mathrm{W}$. Combination of both piezoelectric and electromagnetic able to increase the power output of the system with $14.85 \mu \mathrm{W}$.

\section{References}

1. H. C. Kwang, Sun Power. Kuala Lumpur: Viz Urbana Sdn Bhd (2014)

2. D. Stuart, Thermoelectric Power Generators or Seebeck Power Generation. (online) http://www.electrical4u.com/thermoelectric-power-generators-or-seebeck - powergeneration/ (31 November 2015) (2015)

3. M. El-Hami, P. Glynne-Jones, N.M. White, M. Hill, S. Beeby, E. James, A.D. Brown, J.N. Ross, Design and fabrication of a new vibration-based electromechanical power generator. Sensors and Actuators A: Physical, 92(1), pp.335-342 (2001)

4. M. Duffy, D. Carroll, Electromagnetic generators for power harvesting. In Power Electronics Specialists Conference, 2004. 2004 IEEE 35th Annual, 3, pp. 2075-2081. IEEE (2004)

5. M.F. Hassan, A.R. Yusoff, M.H. Mansor, M.T.C. Kar, Parametric Study of an Electromagnetic Energy Harvester. Applied Mechanics and Materials, 471, p.113 (2013)

6. M.S. Soliman, E.M. Abdel-Rahman, E.F. El-Saadany, R.R. Mansour, A design procedure for wideband micropower generators. Journal of Microelectromechanical Systems, 18(6), pp.1288-1299 (2009)

7. N.M White, P. Glynne-Jones, S.P. Beeby, A novel thick-film piezoelectric microgenerator. Smart Materials and Structures, 10(4), p.850(2001)

8. S. Roundy, On the effectiveness of vibration-based energy harvesting.Journal of intelligent material systems and structures, 16(10), pp.809-823 (2005)

9. H. Yu, J. Zhou, X. Yi, H. Wu, W. Wang, A hybrid micro vibration energy harvester with power management circuit. Microelectronic Engineering, 131, pp.36-42 (2015)

10. V.R. Challa, M.G. Prasad, Y. Shi, F.T. Fisher, A vibration energy harvesting device with bidirectional resonance frequency tunability. Smart Materials and Structures, 17(1), p.015035 (2008)

11. S.P. Beeby, R.N. Torah, M.J. Tudor, P. Glynne-Jones, T. O'Donnell, C.R. Saha, S. Roy, A micro electromagnetic generator for vibration energy harvesting. Journal of Micromechanics and microengineering, 17(7), p.1257 (2007)

12. N.N. Ching, H.Y. Wong, W.J. Li, P.H. Leong, Z. Wen, A laser-micromachined vibrational to electrical power transducer for wireless sensing systems. In Transducers' 01 Eurosensors XV (pp. 38-41) (2001)

13. M.A. Karami, Micro-scale and nonlinear vibrational energy harvesting $\mathrm{PhD}$ Thesis, Virginia Polytechnic Institute and State University (2012) 